\title{
ANTITUMOR AND GENOTOXIC EFFECTS OF LACTOFERRIN IN WALKER-256 TUMOR-BEARING RATS
}

\author{
V.F. Chekhun, D.M. Storchai*, I.N. Todor, T.V. Borikun, N.Yu. Lukianova \\ R.E. Kavetsky Institute of Experimental Pathology, Oncology and Radiobiology, \\ NAS of Ukraine, Kyiv 03022, Ukraine
}

\begin{abstract}
Aim: To investigate the influence of exogenous lactoferrin (LF) on tumor growth, energy and lipid metabolism of Walker-256 carcinosarcoma and to assess genotoxic effects of LF. Materials and Methods: The study was performed on Walker-256 tumor-bearing rats. Total lipids and phospholipids were determined by thin-layer chromatography. Comet assay was used to investigate the genotoxic effects of LF. Results: Daily i.p. administrations of exogenous LF at concentrations of $1 \mathrm{mg} / \mathrm{kg} \mathrm{and} 10 \mathrm{mg} / \mathrm{kg} \mathrm{starting} \mathrm{from}$ the $4^{\text {th }}$ day after tumor transplantation suppressed growth of Walker-256 carcinosarcoma by almost $44 \%$. After treatment with recombinant $\mathrm{LF}$ in both doses, the phospholipid composition of Walker-256 carcinosarcoma cells was changed (3-fold increase of phosphatidylethanolamine, 3.4-fold increase of phosphatidylcholine, and 1.8-fold increase of sphingomyelin, while the cardiolipin content decreased by $67 \%$. Exogenous LF was not genotoxic for bone marrow cells (as assessed by the ratio of PCE/NCE, number of micronuclei) and peripheral blood lymphocytes (percentage of DNA in the tail of a comet) in Walker-256 carcinosarcoma-bearing rats. Conclusion: Exogenous LF caused the inhibition of Walker-256 carcinosarcoma growth and a decrease in the microviscosity of plasma cell membranes, and exerted no genotoxicity toward bone marrow cells and peripheral blood of experimental animals. Key Words: lactoferrin, breast cancer, Walker-256 carcinosarcoma, phospholipid content, genotoxicity.
\end{abstract}

Breast cancer $(\mathrm{BC})$ is one of the most common forms of cancer in women worldwide [1]. Despite the rapid development of modern oncology, the incidence of $B C$ in Ukraine and in most developed countries continues to grow, which makes this problem extremely relevant [1-3]. One of the causes of high mortality from $B C$ is the high heterogeneity of this disease, both in morphological and molecular characteristics, and in clinical course [4-6], which requires different approaches to diagnosis and treatment. Data from clinical and experimental studies confirm the importance of iron metabolism disorders in the onset and progression of cancer [7, 8]. Today, considerable attention is paid to metal-containing proteins, which are important components of iron metabolism and play a role in malignant transformation and tumor progression. There is evidence that the concentration of iron and proteins involved in iron metabolism correlates with the aggressiveness of a number of tumors, including BC $[9,10]$.

Human lactoferrin (LF) is an $80-\mathrm{kDa}$ glycoprotein from the family of iron-binding proteins. It is a single chain polypeptide composed of 692 amino acids. This protein is produced by epithelial cells and is found in mucosal secretions such as tears, saliva, nasal exudates, gastrointestinal fluids, seminal, and vaginal fluids [11]. Synthesis and secretion of LF can occur constitutionally (e.g., in secretory glands) or under the control of sex hormones. It has been described that LF can suppress the growth of solid tumors and strongly inhibit experimental metastasizing in mice [12].

Submitted: August 17, 2018.

*Correspondence: E-mail: dariastorchay@gmail.com Abbreviations used: $\mathrm{BC}$ - breast cancer; BM - bone marrow; LF - lactoferrin; MN - micronuclei; NCE - normochromatophilic erythrocytes; PCE - polychromatophilic erythrocytes.
An important feature of LF is its ability to bind to nucleic acids and to regulate cell cycle, proliferative activity of cells, and to affect the pro- and antioxidant balance in the cells. Human LF is a multifunctional glycoprotein that can inhibit cancer cells at G2/M phase [11, 13].

The present study is focused on the effects of recombinant human LF on tumor growth, energy and lipid metabolism of Walker-256 carcinosarcoma cells in vivo.

\section{MATERIALS AND METHODS}

Design of the in vivo experiment. The study was conducted on female rats $(\mathrm{N}=30)$ with transplanted Walker-256 carcinosarcoma. This tumor strain was obtained from National Bank of Cell Lines and Transplanted Tumors of R.E. Kavetsky Institute of Experimental Pathology, Oncology and Radiobiology (IEPOR), National Academy of Sciences (NAS) of Ukraine (Kyiv, Ukraine). The keeping of animals, all stages of experimental researches, including euthanasia, met the conventional international requirements of humane treatment of laboratory animals, and regulations approved by bioethics commission of the R.E. Kavetsky IEPOR, NAS of Ukraine.

Three groups ( 10 animals each) were formed: 1 - control, 2 - animals treated with exogenous $\mathrm{LF}$ at a dose of $1 \mathrm{mg} / \mathrm{kg}, 3$ - animals treated with exogenous LF at a dose of $10 \mathrm{mg} / \mathrm{kg}$ of body weight. Daily i.p. administration of exogenous LF started from the 4th day after tumor transplantation.

Twelve days after the tumor transplantation, the antitumor activity of LF, the phospholipid composition of tumor cell membranes and the genotoxic effects of LF were analyzed.

Tumor volume was calculated by the formula:

$$
V=a \cdot b \cdot c \cdot \pi / 6
$$

where $a, b, c-$ orthogonal parameters of tumor. 
Percentage of tumor growth inhibition was determined by the formula:

$$
\begin{gathered}
\text { Inhibition } \%=(\text { Control }- \text { Experiment }) / \\
\text { Control } \cdot 100 \%,
\end{gathered}
$$

where Control and Experiment are indicators of tumor volume in the control and experimental groups of animals.

Mitochondria study. Oxidative phosphorylation indices of tumor cell mitochondria were determined according to [14].

Thin-layer chromatography. The isolation of plasma membranes from the tumor cells of rats was carried out by the method of differential ultracentrifugation in sucrose density gradient [15]. Concentration of protein in suspension of plasma membranes was determined by Lowry method. The purity of membrane specimens was controlled by the activity of marker enzymes $\mathrm{Na}^{+}, \mathrm{K}^{+}$-ATPase and 5'- nucleotidase.

Total lipid content in membranes of Walker-256 carcinosarcoma cells (phospholipids, cholesterol, di- and triglycerides and ethers of cholesterol) has been determined. In addition, the following individual phospholipids were evaluated: lysophosphatidylcholine, sphingomyelin, phosphatidylserine, phosphatidylinositol, phosphatidylcholine, phosphatidylethanolamine, cardiolipin.

The qualitative and quantitative composition of total lipids and phospholipids was determined by thinlayer chromatography method using plates "Sorbfil" PTSH-AF-A ("Imid Ltd", Krasnodar, Russia) according to standard procedures [16]. The lipids from the Walker-256 tissue were extracted using a chloroform/ methanol mixture $(1: 1, v / v)$. The thin-layer chromatography of total lipids was carried out only in one direction in the hexane/diethyl ether/glacial acetic acid solvent system (85:15:1, v/v) [16]. The thin-layer chromatography of phospholipids was carried out in two mutually perpendicular directions [17]. The first solvent system was chloroform/methanol/benzene/ ammonia (65:30:10:6, v/v). The second system was chloroform/methanol/benzene/acetone/glacial acetic acid/water (70:30:10:5:4:1, v/v). After the evaporation of the solvents the plates were treated with $10 \%$ $\mathrm{H}_{2} \mathrm{SO}_{4}$ in methanol and heated for $5 \mathrm{~min}$ at $180^{\circ} \mathrm{C}$. All chromatograms were scanned and scans were imaged with the program Picture J. Lipid content was expressed in $\mu \mathrm{gP} / \mathrm{g}$ of the tissue.

Comet assay. A standard classical method of alkaline gel electrophoresis of isolated cells was performed as described in [13]. Microscopic analysis was performed using a fluorescent microscope Axio Scope A. 1 (Carl Zeiss, Germany). For each analyzed specimen at least 100 "DNA-comets" were used. Digital images were analyzed by the computer program "CometScore" (TriTek Corp., USA).

Calculations of micronuclei (MN) were performed on a Axio Scope A. 1 (Carl Zeiss, Germany) fluorescence microscope with magnification $x 400$ by determining the number of cells with MN per 1000 polychromatophilic erythrocytes (PCE). In addition, the ratio of PCE to nor- mochromatophilic erythrocytes (NCE) as an indicator of the cytotoxic effect (per 2000 cells) was evaluated. Following acridine orange staining, PCE had an orange color, NCE - green, and MN - bright yellow. It should be noted that the MN, which were detected in the NCE, were stained in light green.

Statistical analysis. Experimental data were analyzed using the Student's $t$-test. $p$-values less than 0.05 were considered statistically significant. Statisti$\mathrm{cal}$ analysis of the obtained data was performed using the STATISTICA 6.0 software.

\section{RESULTS AND DISCUSSION}

Numerous in vitro and in vivo studies have shown that LF is capable of inhibiting carcinogenesis in the mammary gland, esophagus, lungs and liver [18-21]. Thus, it has been shown that bovine LF is able to reduce proliferative activity and migration of HS578T and T47D cells [18]. The administration of LF in the animal diet led to the inhibition of the development of papillomavirus in rats, as well as adenomas and carcinomas in the lungs of rats, which were treated sequentially with diethylnitrosamine (DEN, i.p.), dihydroxy-di-Npropylnitrosamine (DHPN, in drinking water) and $\mathrm{N}$ nitrosomethylbenzylamine (NMBA, s.c.) during the first 8 weeks [19] and showed chemopreventive action against tongue carcinogenesis initiated with 4-nitroquinoline 1-oxide (4-NQO) in male F344 rats [20].

According to our results, LF administered at a dose of $10 \mathrm{mg} / \mathrm{kg}$ of body weight inhibited the growth of Walker-256 carcinosarcoma by $44.5 \%(p<0.05)$ compared with that in the control group (Fig. 1). A similar result was obtained with the use of LF at a dose of $1 \mathrm{mg} / \mathrm{kg}$, which led to tumor growth retardation by $43.6 \%(p<0.05)$.

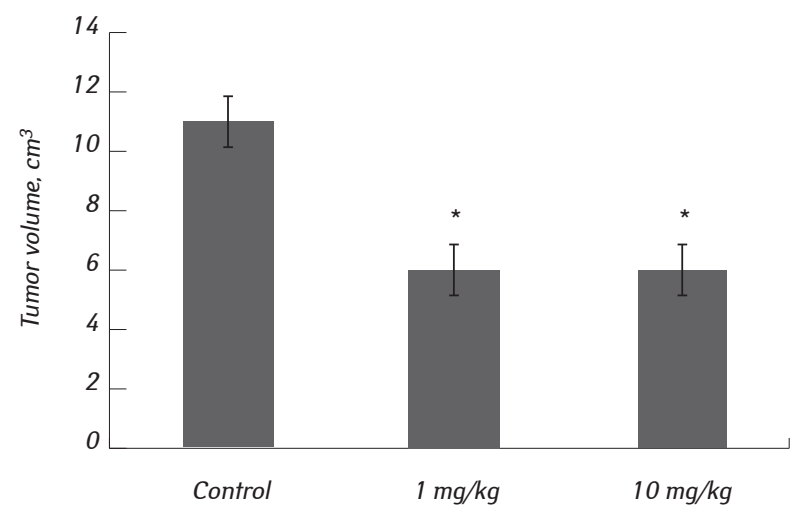

Fig. 1. Effect of exogenous LF on the growth of Walker-256 carcinosarcoma. ${ }^{*} p<0.05$ compared to the control

One of the causes for tumor growth retardation after 9 injections of exogenous LF at the doses of $1 \mathrm{mg} / \mathrm{kg}$ and $10 \mathrm{mg} / \mathrm{kg}$ of body weight may be the suppression of oxidative phosphorylation of the mitochondria in the Walker-256 carcinosarcoma cells. The results of the study are presented in Fig. 2.

As it is evident from the data, exogenous LF administered at doses of $1 \mathrm{mg} / \mathrm{kg}$ and $10 \mathrm{mg} / \mathrm{kg}$ of body weight led to a decrease in the rate of oxygen uptake by mitochondria in the phosphorylating state, that 


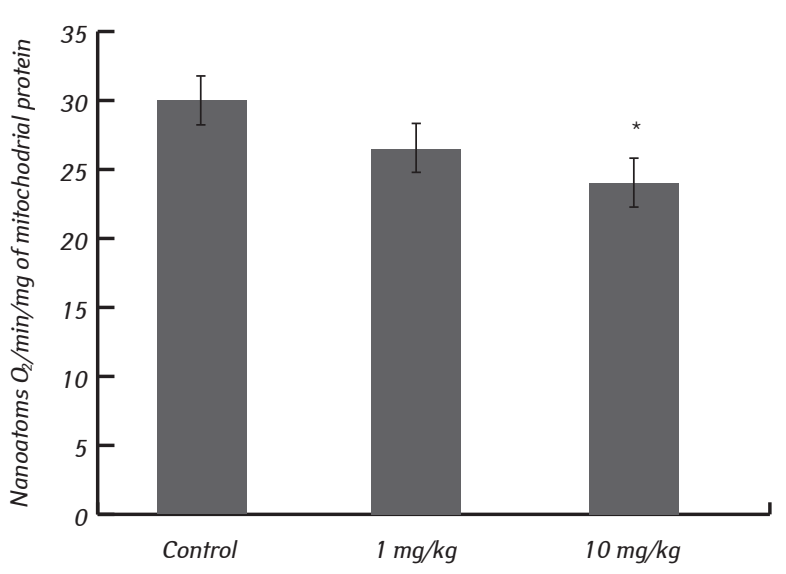

Fig. 2. Effect of LF on the rate of oxygen consumption by the mitochondria of the Walker-256 carcinosarcoma cells in the phosphorylating state (indicator $\mathrm{V}_{3}$ ), the oxidation substrate is glutamate; $\mathrm{n}=10 ;{ }^{\star} p<0.05$ compared to the control

is, during the synthesis of ATP $\left(\mathrm{V}_{3}\right.$ index $)$, by $13 \%$ and $20 \%$, respectively $(p \leqslant 0.05)$. Moreover, such changes were observed only during oxidation of glutamate (NAD-dependent substrate), but not recorded during oxidation of succinate (FADdependent substrate). This indicates that LF is likely

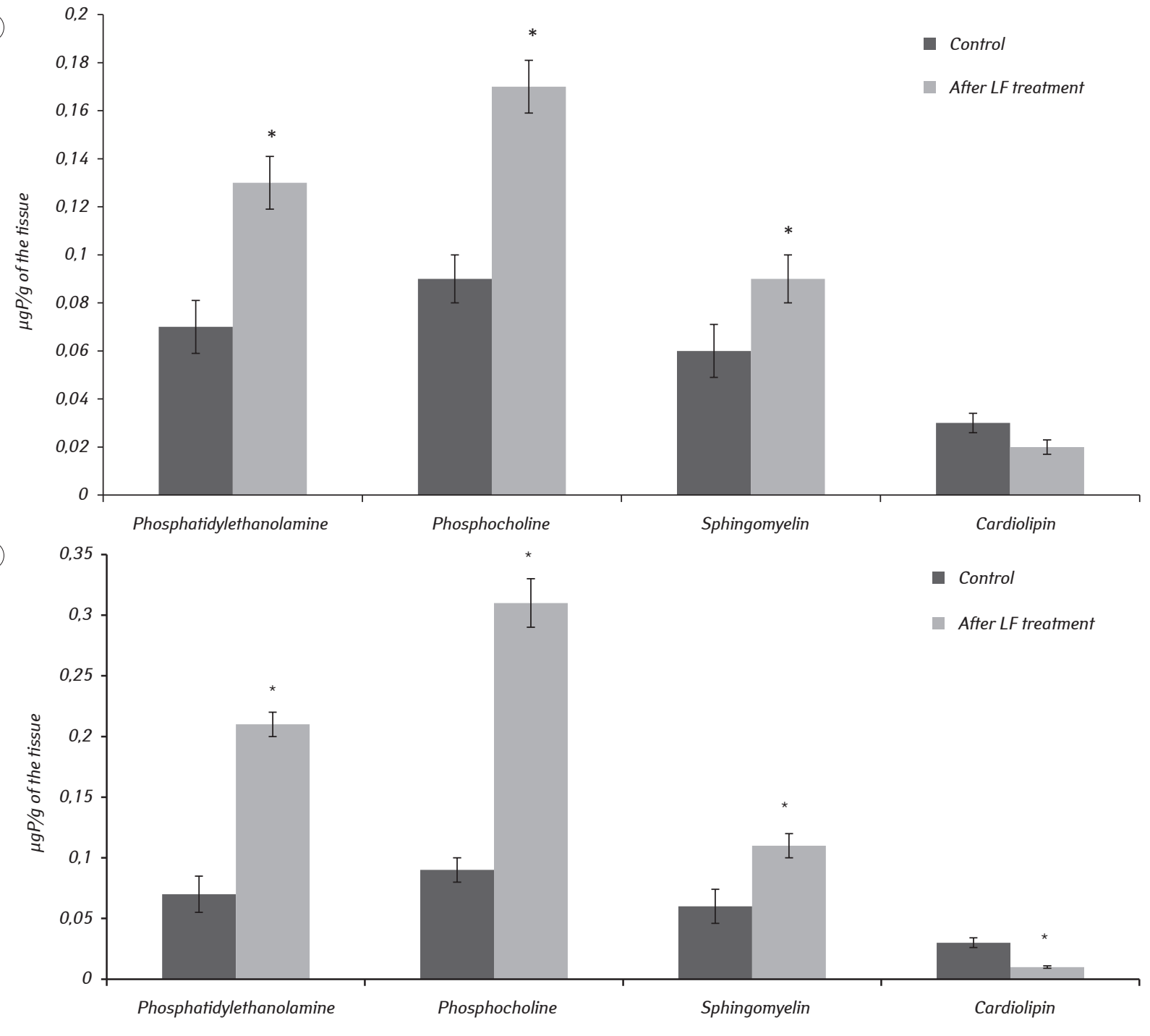

to affect only the first Fe-S complex of the mitochondrial respiratory chain.

It is known that for the normal passage of the cell cycle the anabolism of the lipids of the membranes should be increased, which is accompanied by a change in their architecture. The production of phospholipids, which are essential for cell growth, occurs at different stages of the cell cycle.

Growth and proliferation of cells require certain integrity of the plasma membranes and membranes of organelles. The composition of specific lipids, which form membranes of cells and organelles in proliferating cells, is important for cellular homeostasis. For example, the relative content of key components of membranes, such as phosphatidyl ethanolamine and phosphatidylcholine, are necessary for the optimal functioning of the endoplasmic reticulum $[22,23]$.

Therefore, we investigated the effect of LF on the phospholipid composition of Walker-256 carcinosarcoma cells. The results are shown in Fig. 3. As can be seen from the data shown in Fig. 3, a, nine injections of LF at a dose of $1 \mathrm{mg} / \mathrm{kg}$ led to a significant increase in phosphatidylethanolamine, phosphatidylcholine and sphingomyelin - by $86 ; 89$ and $50 \%$, respec(a) (a)

Fig. 3. Effect of LF on the phospholipid composition of Walker-256 carcinosarcoma cells $(n=9): a-9$ injections of LF in a dose of $1 \mathrm{mg} / \mathrm{kg} ; b-9$ injections of LF in a dose of $10 \mathrm{mg} / \mathrm{kg} .{ }^{*} p<0.05$ compared to control 
tively. Against this background, there was observed a tendency to the decrease of the cardiolipin content (by $33 \%$ ).

At a dose of $10 \mathrm{mg} / \mathrm{kg}$, the content of phospholipids underwent even more evident change (Fig. $3, b$ ). The level of phosphatidyl ethanolamine increased by 3 times, phosphatidylcholine - increased by 3.4 times, sphingomyelin - by 1.8 times. Again, it is worth paying attention to the reduction of the cardiolipin content (by 67\%) (Fig. $3, b$ ). It is supposed that this phospholipid can integrate elements of the respiratory chain of mitochondria into an integral structure, which, in turn, includes the $1^{\text {st }}$ Fe-S complex [23]. In addition, it is believed that before the beginning of apoptosis the interaction of cardiolipin with cytochrome $c$ is disturbed, which may change the integrity of the mitochondrial membrane [23].

However, in our opinion, the most important thing is that LF has led to an increase in the phosphatidylcholine/sphingomyelin ratio (Fig. 4).
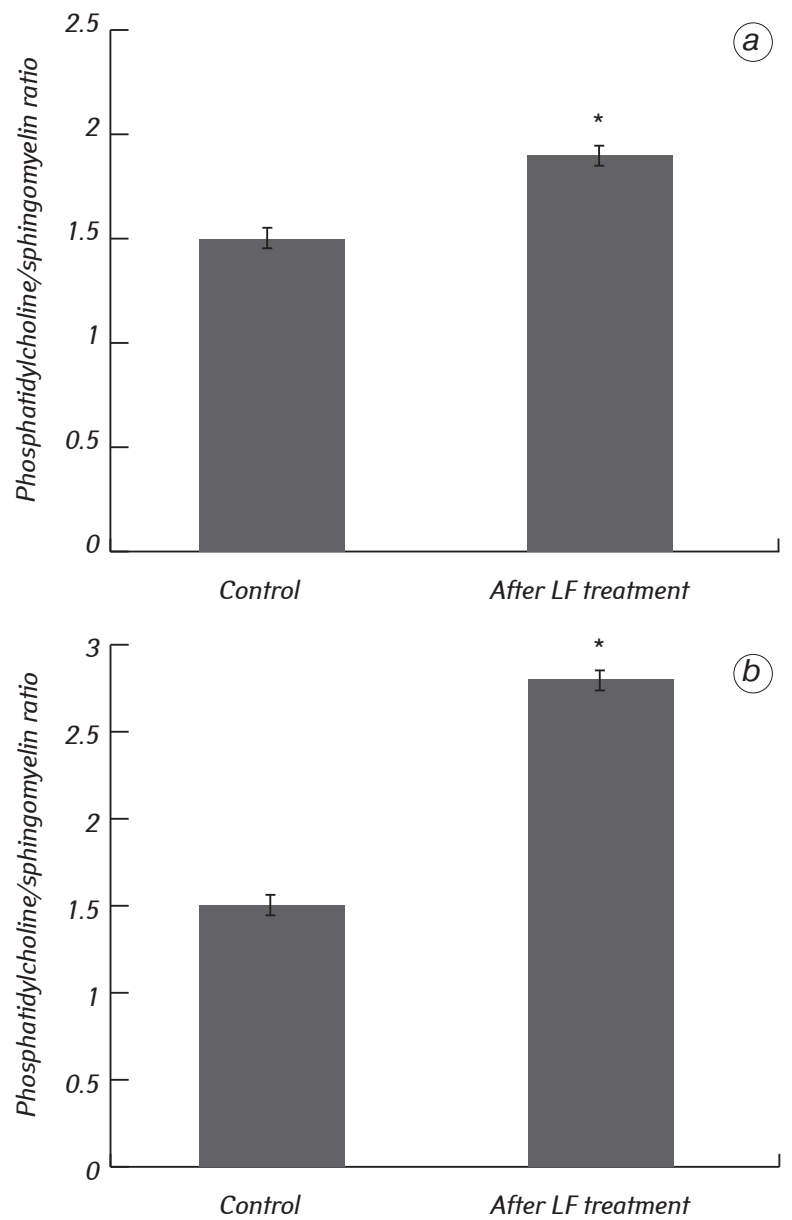

Fig. 4. Effect of LF on the phosphatidylcholine/sphingomyelin ratio in Walker-256 carcinocarcinoma cells $(n=9): a-9$ injections of LF in a dose of $1 \mathrm{mg} / \mathrm{kg} ; b-9$ injections of LF in a dose of $10 \mathrm{mg} / \mathrm{kg}$. ${ }^{*} p<0.05$ compared to control

At a dose of $1 \mathrm{mg} / \mathrm{kg}$, this ratio increased by $27 \%$, and when LF was administered at a dose of $10 \mathrm{mg} / \mathrm{kg}$, this index increased by $87 \%(p<0.05)$. This indicates a decrease in the microviscosity and an increase in the fluidity of the plasma membranes of cells, which leads to changes in the compartmentalization of biologically active molecules in the cell. As a consequence, the cell cannot continue to function normally and to proliferate.
The literature data indicate that the classical method for determining the genotoxic effects of various factors on living organisms is the Hayashi method, which is based on the study of changes in the PCE to NCE ratio in bone marrow (BM). The shift of the $\mathrm{PCE} / \mathrm{NCE}$ ratio in the BM is an indicator of cytotoxic action, and an increase in the $\mathrm{MN}$ number - of genotoxic one [24, 25]. In a number of reports, an attention has been paid to a certain complexity in the evaluation of MN in the cells of the erythroid lineage, which is associated with the specificity of maturation of these cells, namely, the "removal" of the nucleus from the erythroblast, the delay of certain nucleus fragments, followed by their elimination [26]. That is why the quantitative definition of $\mathrm{MN}$ is proposed to be carried out in PCE. We have used the MN test in BM cells only, since, according to some authors, counting $\mathrm{MN}$ and the PCE/NCE ratio in peripheral blood has several disadvantages: Iow PCE content and the possibility of MN elimination in NCE $[27,28]$.

Despite the information on the wide range of LF effects on various parts of the pathophysiological process in carcinogenesis, in literature there is virtually no study of possible mechanisms of toxic effects of LF in vivo. It is believed that an increasing load of "free" iron can take part in the mechanism of cytotoxicity and genotoxicity during carcinogenesis, which in turn may be changed under the action of exogenous iron-binding protein LF. Using the model of Walker-256 carcinosarcoma, we showed that LF at a concentration of $1 \mathrm{mg} / \mathrm{kg}$ did not exert the genotoxic and cytotoxic effects on the BM cells of erythroid lineage $(p>0.01)$ compared with the control group (Table 1).

Table 1. MN (\%o) and the PCE/NCE ratio in BM of animals with

Walker-256 carcinosarcoma under the influence of different concentrations of exogenous LF

\begin{tabular}{lcc}
\hline \multicolumn{1}{c}{ LF dose } & PCE/NCE & MN, \% \\
\hline Control & $0.99 \pm 0.03$ & $0.50 \pm 0.25$ \\
$1 \mathrm{mg} / \mathrm{kg}$ & $0.93 \pm 0.02$ & $1.01 \pm 0.25$ \\
$10 \mathrm{mg} / \mathrm{kg}$ & $0.82 \pm 0.03$ & $1.25 \pm 0.20$ \\
\hline
\end{tabular}

Along with this, LFat a concentration of $10 \mathrm{mg} / \mathrm{kg}$ also did not lead to significant changes in the amount of $\mathrm{MN}$ and the PCE/NCE ratio (Table 1). There is a slight tendency to the shift of the PCE/NCE ratio towards immature erythrocytes in animals with Walker256 carcinosarcoma after the introduction of exogenous LF at a dose of $10 \mathrm{mg} / \mathrm{kg}$, which suggests that LF may insignificantly inhibit erythropoiesis. However, as can be seen from the data, the average values of the $\mathrm{PCE} / \mathrm{NCE}$ ratio in the animals treated with the maximum concentration of $L F$ remained within the control values. Thus, the study of the possible genotoxic and cytotoxic effects of exogenous LF in the group of animals with Walker-256 carcinosarcoma did not reveal any significant changes in the morphology of the BM cells of the erythroid lineage.

In some experimental studies in vitro, it has been shown that one of the possible mechanisms of genotoxic action of LF can be double-strand DNA breaks due to the increased phosphorylation of histone 
H2A.X. However, we have shown that the used concentrations of LF did not cause the genome instability in the MB cells of the erythroid lineage in animals with Walker-256 carcinosarcoma (Table 2).

Table 2. Effect of exogenous LF on peripheral blood lymphocytes in rats with Walker-256 carcinosarcoma

\begin{tabular}{lc}
\hline \multicolumn{1}{c}{ LF dose } & DNA in the tail of the comets, \% \\
\hline Positive control $\left(+\mathrm{H}_{2} \mathrm{O}_{2}\right)$ & $47.3 \pm 4.2$ \\
Control & $5.3 \pm 0.7$ \\
$1 \mathrm{mg} / \mathrm{kg}$ & $6.9 \pm 0.5$ \\
$10 \mathrm{mg} / \mathrm{kg}$ & $6.8 \pm 0.4$ \\
\hline
\end{tabular}

As can be seen from the data presented, the percentage of DNA in the tail of the comets did not exceed significantly the control values at both concentrations of exogenous LF.

In conclusion, the obtained data showed that LF in vivo did not exert genotoxic and cytotoxic effects, as evidenced by the results of a study of BM and the percentage of DNA in the tail of the comet. This indicates the need for further study of the molecular biological effects and mechanisms of the influence of exogenous LF and points to the prospect of its use in clinical practice.

\section{REFERENCES}

1. Siegel RL, Miller KD, Jemal A. Cancer statistics. CA: a Cancer Journal for Clinicians 2018; 68: 7-30.

2. Siegel RL, Miller KD, Jemal A. Cancer statistics. CA: a Cancer Journal for Clinicians 2016; 66: 7-30.

3. Postevka ID. Current state of the problem of breast cancer screening (review of the references). Buk Med Herald 2016; 20: 199-203 (in Ukrainian).

4. Nahid AA, Kong Y. Involvement of machine learning for breast cancer image classification: a survey. Computational and Mathematical Methods in Medicine 2017; 2017: 1-29.

5. Turashvili G, Brogi E. Tumor heterogeneity in breast cancer. Front Med 2017; 4: 227.

6. Chekhun VF, Zalutskii IV, Naleskina LA, et al. Modifying effects of lactoferrin in vitro on molecular phenotype of human breast cancer cells. Exp Oncol 2015; 37: 181-6.

7. Torti SV, Torti FM. Iron and cancer: more ore to be mined. Nature Rev Cancer 2013; 13: 342.

8. Konstorum A, Lynch ML, Torti, SV, et al. A systems biology approach to understanding the pathophysiology of high-grade serous ovarian cancer: focus on iron and fatty acid metabolism. OMICS 2018; 22: 502-13.

9. Torti SV, Torti FM. Cellular iron metabolism in prognosis and therapy of breast cancer. Criti Rev Oncog 2013; 18: $435-48$.

10. Chekhun VF, Lukyanova NY, Burlaka AP, et al. Iron metabolism disturbances in the MCF-7 human breast cancer cells with acquired resistance to doxorubicin and cisplatin. Int J Oncol 2013; 43: 1481-6.

11. Li WY, Li QW, Han ZS, et al. Growth suppression effects of recombinant adenovirus expressing human lactoferrin on cervical cancer in vitro and in vivo. Cancer Biother Radiopharm 2011; 26: 477-83.

12. Bezault J, Bhimani R, Wiprovnick J, Furmanski P. Human lactoferrin inhibits growth of solid tumors and development of experimental metastases in mice. Cancer Res 1994; 54: $2310-2$.
13. Zalutskii IV, Lukianova NY, Storchai DM, et al. Influence of exogenous lactoferrin on the oxidant/antioxidant balance and molecular profile of hormone receptor-positive and-negative human breast cancer cells in vitro. Exp Oncol 2017; 39: 106-11.

14. Todor IN, Chekhun VF. The respiration of mitochondria from liver of rats treated with cisplatinum and millimeter range electromagnetic radiation. Exp Oncol 1995; 17: 137-40.

15. van Amelsvoort JM, Jansen JW, De Pont JJ, Bonting SL. Is there a plasma membrane-located anion-sensitive ATPase? IV. Distribution of the enzyme in rat pancreas. Biochim Biophys Acta 1978; 512: 296-308.

16. Todor IN, Lukyanova NY, Chekhun VF. The lipid content of cisplatin-and doxorubicin-resistant MCF-7 human breast cancer cells. Exp Oncol 2012; 34: 97-100.

17. Vaskovsky VE, Terekhova TA. HPTLC of phospholipid mixtures containing phosphatidylglycerol. J High Resolut Chrom CC 1979; 2: 671-2.

18. Duarte DC, Nicolau A, Teixeira JA, et al. The effect of bovine milk lactoferrin on human breast cancer cell lines. J Dairy Sci 2011; 94: 66-76.

19. Ushida Y, Sekine K, Kuhara T, et al. Possible chemopreventive effects of bovine lactoferrin on esophagus and lung carcinogenesis in the rat. Jpn J Cancer Res 1999; 90: $262-7$.

20. Tanaka T, Kawabata K, Kohno H, et al. Chemopreventive effect of bovine lactoferrin on 4-nitroquinoline 1-oxide induced tongue carcinogenesis in male F344 rats. Jpn J Cancer Res 2000; 91: 25-33.

21. Fujita K-i, Ohnishi T, Sekine K, et al. Down-regulation of 2-Amino-3,8-dimethylimidazo[4,5-f]quinoxaline (MeIQx)-induced CYP1A2 expression is associated with bovine lactoferrin inhibition of MeIQx-induced liver and colon carcinogenesis in rats. Cancer Sci 2002; 93: 616-25.

22. Sanchez-Alvarez M, Zhang Q, Finger F, et al. Cell cycle progression is an essential regulatory component of phospholipid metabolism and membrane homeostasis. Open Biol 2015; 5: 150093.

23. Gula NM, Margitich VM. Fatty Acids and Their Derivatives in Pathologic States. Kyiv: Naukova Dumka, 2009. 334 p. (in Ukrainian).

24. Suzuki H, Ikeda N, Kobayashi K, et al. Evaluation of liver and peripheral blood micronucleus assays with 9 chemicals using young rats: A study by the Collaborative Study Group for the Micronucleus Test (CSGMT)/Japanese Environmental Mutagen Society (JEMS) - Mammalian Mutagenicity Study Group (MMS). Mutation Res/Genetic Toxicol Environm Mutagen 2005; 583: 133-45.

25. Celik A, Öğenler O, Çömelekoğlu Ü. The evaluation of micronucleus freguenc by acridine orange fluorescent staining in peripheral blood of rats treated with lead acetate. Mutagenesis 2005; 20: 411-5.

26. Nersesyan AK. Biomonitoring of cytogenetic effect of antitumor therapy by means of micronucleus assay in exfoliated epithelial cells. Tsitol Genet 2007; 6: 67-73 (in Russian).

27. Kovaleva OA. Cytogenetic anomalies in somatic cells of mammals. Tsitol Genet 2008; 1: 58-72 (in Russian).

28. Firsova SS, Bucharskaya AB, Maslyakova GN, et al. The changes of morphological indicators of bone marrow and peripheral blood at long exposure of gold nanoparticles. Izvestiya Saratov University 2011; 11: 54-7 (in Russian). 